\title{
Practical guidance for healthcare professionals during the COVID-19 pandemic; with special emphasis on the care of cardiovascular patients and personal protection
}

\author{
Chugh Y. ${ }^{1}$, Chugh S. ${ }^{2}$, Kumar Chugh S. ${ }^{3 *}$ \\ DOI: https://doi.org/10.17511/ijmrr.2020.i03.11 \\ ${ }^{1}$ Yashasvi Chugh, Chief Fellow, Cardiovascular Medicine, Icahn School of Medicine, Mount Sinai and St. Luke`s Hospital, New York, USA. \\ 2 Sunita Chugh, Consultant, Non-Invasive Cardiology, Jaipur National University Hospital and Medical College, Institute for Medical Sciences \\ and Research Center, Jaipur, Rajasthan, India. \\ 3* Sanjay Kumar Chugh, Head of Department of Cardiology and Interventional Cardiology, Jaipur National University Hospital and Medical \\ College, Institute for Medical Sciences and Research Center, Jaipur, Rajasthan, India.
}

A novel, severe acute respiratory syndrome coronavirus 2 (SARS-CoV-2) is causing the COVID-19 pandemic. There are concerns regarding the spread of disease by asymptomatic carriers to healthcare workers who continue to see patients and to perform procedures on them. The infection then could be transmitted through them to other patients who eventually, could infect persons in the community. Further, limitations of existing tests to detect new cases that are negative early in the disease; and the inability to use appropriate personal protection equipment (PPE)also contribute to the spread of infection. This document, from the COVID-19 Working Group of Wellness and Radial Intervention Society, describes considerations for management and care of cardiovascular disease (CVD) patients with the concept of COVID 19 'designated Vs. enabled' centers for those with symptoms of and/ or confirmed COVID-19 disease Vs. asymptomatic suspects. It also provides guidance to healthcare professionals, hospital administrators, and policymakers in general and to those managing patients with CV and other diseases, regarding the concept of 'levels of personal protection ' for staff; with suggestions for 'equivalent innovative alternatives', and 'a protection plan' for patients to prevent the spread of disease.

Keywords: Coronavirus, COVID-19, Cardiovascular disease, Cardiac catheterization laboratory, Echocardiography, Coronary care unit, Invasive, Semi-invasive, and non-invasive procedures, Personal protection equipment (PPE)

\section{Corresponding Author}

Sanjay Kumar Chugh, Head of Department of Cardiology and Interventional Cardiology, , Jaipur National University Hospital and Medical College, Institute for Medical Sciences and Research Center, Jaipur, Rajasthan, India.

Email: skchughcardiology@yahoo.com

\section{How to Cite this Article}

Chugh Y, Chugh S, Chugh SK. Practical guidance for healthcare professionals during the COVID-19 pandemic; with special emphasis on the care of cardiovascular patients and personal protection. Int J Med Res Rev. 2020;8(3):277-287.

Available From

https://ijmrr.medresearch.in/index.php/ijmrr/article/ view/1179
To Browse

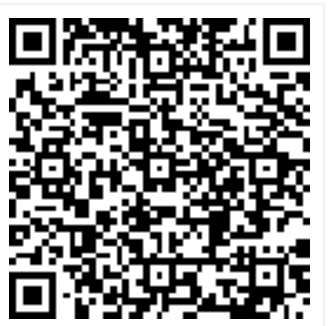

Manuscript Received 2020-04-10

Conflict of Interest No
Review Round 1 2020-04-20

Funding Nil

Review Round 2
2020-04-26
Ethical Approval
Yes

Review Round 3

Accepted 2020-05-02

Plagiarism X-checker $8 \%$

(c) 2020 by Yashasvi Chugh, Sunita Chugh, Sanjay Kumar Chugh and Published by Siddharth Health Research and Social Welfare Society. This is an Open Access article licensed under a Creative Commons Attribution 4.0 International License ttps://creativecommons.org/licenses/by/4.0/ unported [CC BY 4.0]. 


\section{Introduction}

A novel human coronavirus, the severe acute respiratory syndrome coronavirus 2 (SARS-CoV-2) is causing the COVID-19, pandemic $[1,2]$. On account of its high mortality rate, together with the lack of any specific treatment, and the challenge of treating large numbers of patients in hospitals with fear of overwhelming the existing healthcare facilities, efforts are being focused on containing the disease and preventing spread in the community during this COVID-19 pandemic [3].

This document from the working group for COVID19 of Wellness and Radial Intervention Society presents a strategy of triaging patients to different segregated centers and discusses plans for the protection of patients and doctors and other healthcare personnel with special emphasis on the care of cardiovascular patients.

Limitations SARS-Cov-2 Testing: Till recently, in the US, all nasal and throat swabs for reverse transcriptase-polymerase chain reaction ( $R T-P C R$ ) had to be sent to the Centers for Disease Control and Prevention (CDC) as well as to some commercial labs such as Quest Diagnostic (DGX) and Labcorp (LH); all of which required mailing swabs to labs for testing with prolonged turnaround time(TAT). Though the TAT of a rapid molecular point-of-care test by Abbott, Thermo Fisher and Roche for the coronavirus, recently approved by the U.S. Food and Drug Administration is less than 15 minutes; high false negativity in early stages of the disease [4] is still a concern; thereby warranting repeat testing after few days. In India, the TAT for the RT-PCR test is still $48-72$ hours.

In a hospital setting, preventing the spread of infection to health care personnel and thence to other staff and patients is a big challenge because healthcare settings are vulnerable to the introduction and spread of SARS-CoV-2 because of:

- the high rate of its transmission by infected persons, including asymptomatic carriers, who may be visitors, hospital staff, or patients with symptoms unrelated to COVID-19; such as patients with cardiovascular (CV) or other diseases. The asymptomatic carriers are known to shed and transmit the virus from their upper respiratory tract [4]. Of the 634 passengers who tested positive for COVID-19 on the Diamond Princess cruise ship; $17.9 \%$ were asymptomatic [5]. The proportion of pre-symptomatic transmission was $48 \%$ (95\%CI 32-67\%) for
Singapore and $62 \% \quad(95 \%$ CI $50-76 \%)$ for Tianjin, China [6].

- the long incubation period for COVID-19 of up to 14 days, during which time a person may be asymptomatic and yet unknowingly spreading the infection to others.

- the stability of SARS-CoV-2 in aerosols [7] and on surfaces (up to 3 days for plastic) contributes further to the high risk of transmission of the virus

- limitations in diagnostic testing for COVID-19, including the fallacy of an initial negative COVID-19 test report; Further compounded by-

- a lack of understanding of these issues and of the concept of adequate personal protection in this situation by many healthcare personnel, specialist doctors, institutional heads and policymakers worldwide as corroborated by a breaking news item reported by MSNBC on second April 2020, that the Georgia Governor Brian Kemp wasn't aware that asymptomatic people could pass coronavirus. It comes as no surprise, and he definitely is not the only one; many hospital administrators, policymakers, and institutional heads may similarly not be aware of the same.

Existing protocols in many hospitals worldwide still allow hospitals to keep healthcare workers exposed to COVID-19 patients on the job with the stipulation of a daily screening for symptoms. Hence there are concerns regarding the spread of disease to healthcare workers who continue to see patients and to perform procedures on them, thereby risking spread to others. The CV disease (CVD) patients are known to have a higher fatality with COVID 19. As patients with significant cardiovascular risk factors, or established CVD get affected by COVID 19 infection, cases of Acute coronary syndrome (ACS), ST-elevation acute myocardial infarction (STEMI), in the setting of COVID-19 may increase.

Since patients presenting with ACS and STEMI, require immediate admission and often urgent intervention, without allowing time for assessment for COVID 19 status in these patients; this increases the risk of exposure of cardiologists, healthcare personnel and other admitted patients. A low rate of detection of infection in early stages of the disease makes it even more difficult to accurately diagnose the disease early, leading to a dilemma for the cardiologist as to the best course of action for saving the life of such a patient, without compromi- 
Sing the safety of the staff and other admitted patients.

Further, to avoid unnecessarily exposing the cardiac catheterization laboratory (CL) staff to infection, cardiologists should be aware that COVID 19 myocarditis closely mimics electrocardiographic changes of ACS including STEMI and is associated with raised cardiac markers The graph of active newly diagnosed COVID-19 cases per day is steep upwards worldwide (except China) (Figure 1a) as in India (Figure-1b). The rising number of doctors and staff becoming positive for COVID-19 and dying from the disease is also a matter of grave concern. Hence it has become urgently necessary to educate health care personnel in general, regarding care and management of patients who may be in one of 3 categories (i) symptomatic with flu-like symptoms and under investigation (IUE ) or (ii) overtly COVID19 positive or (iii) asymptomatic carriers; with regard to their own protection as well as that of the patients.

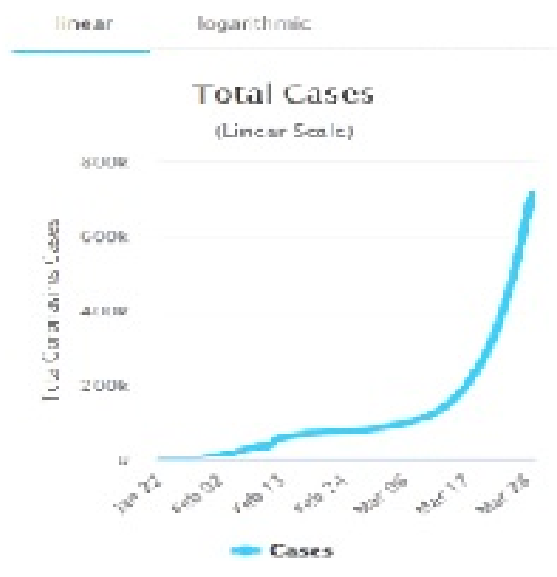

Fig-1b: New COVI19 positive cases per day in India as on 27th March 2020 (From https://www.worldometers.info/coronavirus / , last updated 30th March 2020.

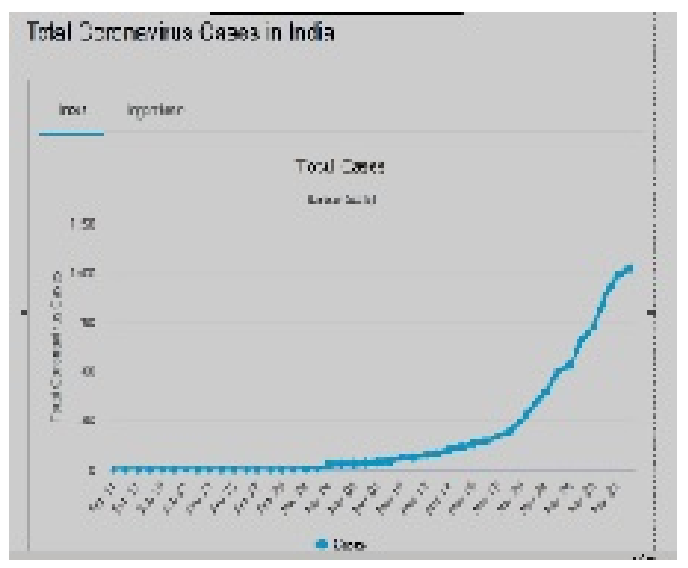

Fig-1a: WHO data on cases of COVID 19 worldwide as on 30th March 2020 (From https: / / www.worldometers.info/coronavirus / , last updated 28 March 2020.

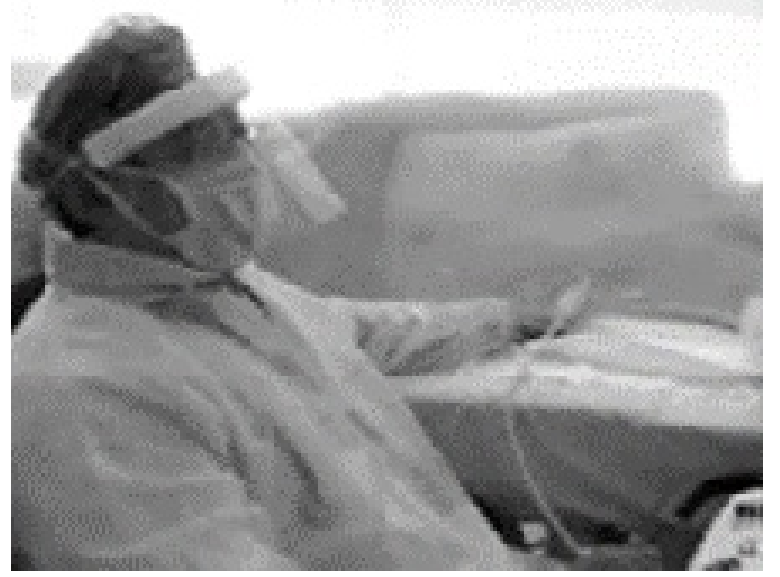

Fig-2. Inadequate Personal protection for operator performing echocardiogram in COVID-19 patient. The illustration shows an echocardiographer with a cap, mask, and face shield with the use of a barrier. This does not constitute appropriate personal protection. Level 1 PPE is mandatory for CCS I or II (see text for details); while Level 2 PP may suffice for CCSI II (see text for details).

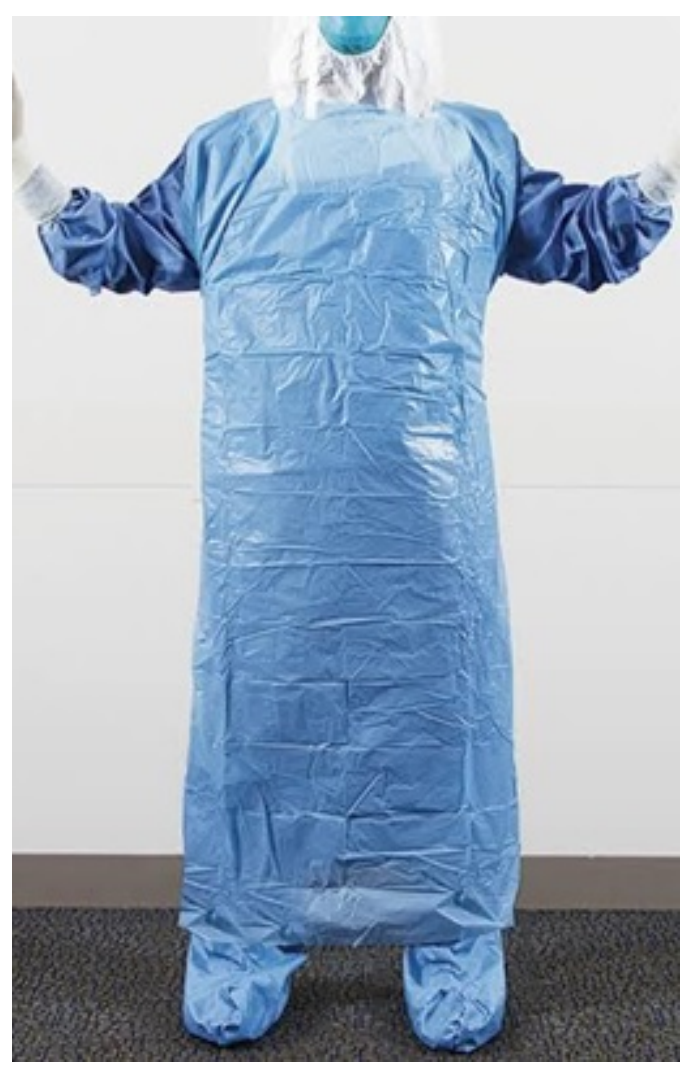

Fig-3: An innovative suite equivalent alternative to a HAZMAT suit. Though it has no 
Rubber. It is made of non-porous fabric and plastic. This could be used to form the 2 nd layer of cover on the body in both Level 1 and 2 PP. (Please see Appendix A and B).

While appropriate personal protection equipment (PPE) is important for every health care personnel; it is more important for them to develop an understanding of the levels of personal protection required during the discharge of their various duties depending on their services (Appendix A-C). If PPE is not available, alternative equivalent arrangements can be and should be made as described in Appendix A and B. It is worrying that health care personnel sometimes work without adequate PP not only because of a lack of knowledge on their part and that of the hospital administrators and policymakers; but also because of non-availability of PPEs, which puts the lives of this health care personnel at risk. Many cardiologists and other specialists performing invasive and interventional procedures wrongly believe that a universal protection kit used for Hepatitis B or HIV positive cases is adequate for combatting the risk of infection from COVID-19. Cardiologists are still enquiring on twitter whether N95 masks should be worn for all cases. This is so because there is no guideline and no paper to guide them. This paper aims to do just that; to disseminate information to guide healthcare workers appropriately. Figure-2 illustrates inappropriate PP for persons performing echocardiogram at one hospital. The PP is not appropriate because it is inadequate, because the virus is transmitted via droplets, is highly contagious and can survive on various surfaces as detailed above; hence three layers of cover on the head, body, and feet is important, together with 2 masks, one of which should be N95. The Government of state of Delhi, India and the Federal Government of India has offered life insurance to those involved at the frontline, managing COVID 19 patients; but were unable to meet the requirement of essential PPEs for all healthcare personnel initially till local production increased to over 4 lakh PPEs per day, at the time of going to press. The same problem was reported by health care personnel on social media worldwide, including from the US and UK. In the US, healthcare personnel has even been penalized by hospitals for publicizing the issue. Hence this paper also suggests the equivalent alternatives (Figure 3) and stresses the need for three levels of cover for various parts of the body of every health care personnel involved in various kinds of services, along with suggested material
(Including at least one additional plastic and one additional non-porous fabric in addition to the usual), to safeguard the personnel from risk of infection from COVID-19 patients and suspects (Appendix A-C).

To recall the words of Charles Darwin 'It is not the fittest who survive, nor the most intelligent; but those who adapt to change'.

The pandemic may abate over next few months; the steep rise of new cases may reach a plateau, but it is reasonable to believe that the disease will remain at varying levels around us for long; hence we need to adapt to change and change our practices accordingly to meet the challenge for years to come. Hence the guidance in this document should be considered a suggestion for a change in practice, to adapt to COVID-19; not just a short- term, stopgap measure for a few weeks. This document, from the COVID-19 Working Group of the Wellness and Radial Intervention Society, also describes considerations for management and care of cardiovascular disease (CVD) patients with the concept of COVID-19 'designated' Vs. 'enabled' centers for those with symptoms of and/ or confirmed COVID-19 disease Vs. asymptomatic suspects. It also provides guidance to healthcare professionals, hospital administrators, and policymakers in general and to those managing patients with $\mathrm{CV}$ and other diseases, regarding the concept of 'levels of personal protection ' for staff; with suggestions for equivalent alternatives, and a' protection plan ' for patients to prevent the spread of disease. The guidance can also be broadly applied to surgical branches in general and to other specialties such as gastroenterology, urology, anesthesiology, otolaryngology, and ophthalmology involved with invasive, semi-invasive, and noninvasive procedures. The latter three specialties carry a higher risk of exposure for healthcare professionals.

\section{Proposal for Triage of all patients countrywide:} The patients presenting to any hospital countrywide should be triaged based on their infection status category, referred to as 'clinical COVID-19 status Category(CCSC)' (see below) and managed in an appropriate center, as described below under B.

\section{A. Clinical COVID 19 status Categories (CCSC)- (Table-1)}

01. Confirmed COVID-19 positive: To be managed in 'COVID-19 designated' centers only 
02. Individual under Evaluation (IUE) as defined by the presence of

- A person who arrived from; or was in contact with another person who arrived from a COVID-19 affected country.

- Fever

- cough, sore throat, breathlessness. To be preferably managed in a 'COVID-19 designated' center.

03. III. Asymptomatic for COVID symptoms; with negative COVID test. Since RT-PCR can be false negative in the early stages of the disease [4], such persons could well be COVID carriers and hence they should be treated in a 'COVID enabled' center. Such patients should be screened first for fever, cough, a CT Chest to rule out lung infiltrates, and an RT-PCR test for antigen [8], and /or IgM and IgG testing if available, and should be accepted for admission to the hospital only if found to be in CCS III.

\section{Table-1: Concept of separate COVID-19 'designated' and 'enabled' centers for managing patients.}

CENTERS OF CARE SHOULD BE SEGREGATED:

DON'TLETHOSPITALS BECOME CENTRES OF SPREAD OF INFECTION

COVID19 'DESIGNATED CENTRES' COVID19 'ENABLED CENTRES'

(For treatment of patients with COVID19) (or NON-COVID centers-For treating NON-COVID illnesses)

- RT-PCR POSITIVE FOR COVID19 - PATIENTS ASYMTOMATIC FOR

INFECTION COVID19; WITH NEGATIVE RT-PCR OR

(40\% MAYBE FALSE NEGATIVE)

- PERSONS UNDER INVESTIGATION FOR
COUGH,FEVER, WITH NONON-COVID ILLNESS
CHOSE WITH

COUGH,FEVER,AND THOSE WITH
CONTACT HISTORY OR TRAVEL TO

CONTACT HISTORY OR TRAVEL TO CARRITR $\&$ DOCRS,NURSES, OTHER PATIENTS
AFFECTED REGIONS

$\rightarrow$ WHICH GOES BACK TO SPREADING

INFECTION TO COMMUNITY

MERIT LEVEL-1 PERSONAL PROTECTION FOR ALL STAFF

MERTS LEVEL 1 PERSONALPROTECTION FOR CCU,CL,\& OR 尔atients

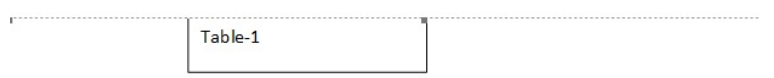

CENTERS OF CARE SHOULD BE SEGREGATED: DON'TLET HOSPITALS BECOME CENTRES OF SPREAD OF INFECTION COVID19 'DESIGNATED CENTRES' For treatment of COVID 19 \&/or any other speciality treatment $\quad$ For any speciality treatment- excluding C

- POSITIVE FOR COVID19 INFECTION OR

- PERSONS UNDER INVESTIGATION FOR COUGH,FEVER,AND THOSE WITH CONTACT HISTORY OR TRAVEL TO AFFECTED REGIONS

- ANY PATIENT COMING FOR TREATMENT UNDER ANY SPECIALIT IS SUSPECT AS THEY MAY BE ASYMTOMATIC CARRIERS

- TESTING THEM DOES NOT HELP IN INITIAL 7-10 DAYS AS THEY MAY BE FALSE NEGATIVE \& RISK INFECTING DOCTORS, NURSES,OTHER PATIENTS $\rightarrow$ WHICH GOES BACK TO SPREADIN INFECTION TO COMMUNITY

MERITS LEVEL-1 PERSONAL PROTECTION FOR ALL STAFF

MERITS LEVEL 2 PERSONALPROTECTION FORAL

Allocation of the center to patients: Two separate types of centers should be available in
Every city, region, or town for managing CVD and other patients, based on their CCSC, as described below. The considerations for other non-cardiology, semi-invasive and invasive procedures (NCSIIP) such as intubation, trans-oesophageal echocardiography, endoscopy, and various ophthalmology and otolaryngology, dental and other miscellaneous procedures, with respect to healthcare personnel and patients, at such centers, would be similar to that for cardiology procedures.

Though such segregated centers were available in China and also in India in Stage 2 of the epidemic; the caveat is that if numbers overwhelm the capability of the existing facility then it may become difficult to maintain such segregation. In India, at the time of going to press, this differentiation is now being lost; since with increasing numbers, several private hospitals in Delhi and Mumbai are catering to both COVID-19 and Non-COVID-19 patients in different wards in the same hospital. This is contrary to what was done in China [9] and endangers the spread of infection, and risks hospitals becoming epicenters of infection and should be discouraged.

Rationale: The rationale for such segregation of centers or hospitals is to minimize the risk of crossinfection and maximize care. This is so because in a designated center, the health care personnel and set up is completely geared to deliver care without delay to all such CCS I and II patients without hesitation, regardless of specialty. Unfortunately, for countries which got overwhelmed by the pandemic such as the USA in stage 4 or Italy and Spain in stage 7 of the epidemic; differentiation into COVID19 designated or enabled centers was not possible as was done initially in India, where these steps were taken in early stages of the epidemic.

If a patient of CCS I or II were to be treated in a hospital shared by all three categories of CCS and if such a patient, for example, were to have a cardiac arrest, then there could be hesitation and delay in the steps of cardiopulmonary resuscitation (CPR), as is observed in the directive issued from the British Resuscitation Council for CPR in suspected or confirmed COVID-19 cases, where the new directive is to delay chest compressions till a " FULL COVID19 PPE is worn. This is so because COVID and nonCOVID hospitals have not been segregated in the UK. In a COVID designated hospital, staff would already be in full PPE; hence this delay would not be necessary. In the USA however, most hospitals have a policy of no CPR in critically ill COVID-19 patients, to avoid exposure to staff. 
Even after the curve of new cases plateaus or starts declining, it is anticipated that the incidence of new cases may remain high for a year or more, with a chance of endemicity. This means that this may be a protracted battle against COVID-19; and therefore the above plan of management should become the norm, for those working in either of the two centers-COVID-19 designated or COVID-19 enabled centers, rather than wait for the curve to plateau or lockdown of cities to end, for regions where lockdown is being done to control the spread of disease. At the present time, there is no clarity to the public regarding demarcation of the two types of centers: one for COVID-19 (COVID-19'designated centers ') and the other for other patients who are asymptomatic suspects; but not proven COVID-19 nor under investigation for COVID-19 (COVID-19 'enabled centers') in the Indian sub-continent. Hence footfall of patients suffering from other NonCOVID illnesses has also fallen. This, combined with a national lockdown of 21 days, which is likely to be extended in order to check the spread of disease, spells disaster for the hundreds of thousands of patients requiring elective and urgent treatment including STEMIs, Non-STEMIs, and strokes, who are keeping away from hospitals for fear of contracting the disease.

Hence not only is it important to separate the care into two types of segregated centers, as above; the same should be advertised and made clear to the people and patients who are currently afraid of visiting hospitals for their ailments.

\section{A) COVID-19 'enabled '(or NON-COVID center) center: For treating patients in CCS III category}

Personal protection (PP) considerations for health care personnel: At such a center, all staff, including physicians, irrespective of their area of work; whether in the out-patients', wards, emergency room, critical care (CCU) areas, $\mathrm{CL}$ or operating room(OR) should have Level 1PPE. In case of non-availability for financial or other reasons, Level -2 personal protection(PP) may be acceptable for personnel not in $\mathrm{CCU}, \mathrm{CL}$ or $\mathrm{OR}$. as described in details in Appendix A. Appendix C (1-3) from CDC pictorially illustrates the steps and precautions for donning and doffing of personal protection equipment (PPE).

Further, general precautions as described in Appendix -B need to be applied for work areas for patients requiring either or both of the following:
01. Non-invasive and conservative management (including $\mathrm{CCU}$ ).

02. Semi-invasive, Invasive and interventional procedures including all procedures in the cardiac catheterization laboratory $(\mathrm{CL})$.

Patient protection policy: Single or isolated room for every single patient is necessary for such patients for initial 5-7 days, following which the patient can be re-screened and tested for the exclusion of COVID-19. Only after COVID-19 is excluded on repeat testing, should the patient be shifted out of the isolation room [8] to the floor or discharged. The policy of social distancing, avoidance of crowding, use of hand-rub for patients and maintenance of hand-hygiene, along with appropriate protective gear including surgical masks [10], caps of non -porous fabric, hospital dress, non-porous gowns, and footwear, and disallowing any contact with visitors must be strictly followed. Visitors should be restricted and should wear masks. All visitors should be screened to exclude CCS categories I and II.

\section{B) COVID-19 'designated' center}

Few centers in every region should be 'designated' for managing COVID-19 positive cases. These centers should be fully- equipped to provide inhospital services in all specialties and have Level-1 PP for all categories of staff (please refer to Appendix A-C for details), in addition to institutional standard operating protocols (SOPs), adequate staffing and a staff roster to match the requirements as discussed in detail below. The choice and number of such centers should be based on the estimated burden of disease per million population in the region which can be decided by the regional authorities. These COVID-19 positive cases should be treated in such designated centers only.

These centers should be geared to provide care in all specialties; including both of the following in Cardiology:

01. conservative care in outpatients, on the floors as well as in coronary care or other intensive care units

02. invasive and interventional management in the cardiac catheterization laboratory $(\mathrm{CL})$ as described below.

\section{A. Plan of Management (POM) for various CCS categories}

Following would be a suggested plan of action 
(POM) for various clinical COVID-19 status categories:

01. CCS Category I and CCS category-II Patients with IUE: should be managed in 'COVID-19 designated centers' with Level 1 PP. (AppendixA-C).

Acute coronary syndrome (ACS), ST-elevation acute myocardial infarction (STEAMI), heart failure, Heart block, and other miscellaneous adult CVD cases not belonging to CCS category I or II should be managed conservatively initially, in a 'COVID 19 enabled ' CCU. This is because the CCS category of some patients might change during the period of initial stabilization and might then need to be referred to a COVID designated center. It takes time for staff and operators to prepare the $\mathrm{CL}$ with precautions and to get ready with adequate protection (Level1) which delays procedures. However, if at any time, their CCS category changes to CCS III; they could be referred to a 'COVID enabled' center with $\mathrm{CL}$ for invasive and interventional management.

Only the following should be shifted urgently to the $C L$, with Level 1 PP (Table- 2) and general precautions (as detailed in Appendix $A$ and $B$ ):

A) Patients requiring urgent percutaneous coronary intervention (PCI) for:

01. NSTEMI with refractory angina,

02. High-risk NSTEMI / STEMI, with one or more of the following: hemodynamic compromise, electrical instability, refractory chest pain,

03. Inferior STEMI with RVMI;

04. Large Anterior STEMI;

05. Rescue PCI

(B) Those requiring urgent implantation of a temporary or permanent pacemaker.

Table-2: The two levels of protection.

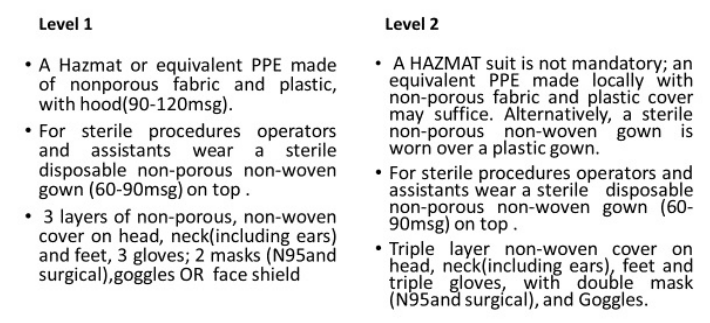

Level of pr Table 2

Level 1

- Level-1 PP

- Includes donnin

clothing on heas

masks(N95and s

and face shield

- A Hazmat or eqi

nonporous fabri

preferably with

sterile disposab

universal protec

It may be preferable to perform invasive and interventional procedures transradially because risk of bleeding [11] and therefore contact time between staff and patients and hence risk of cross -infection may be less with transradial, compared to transfemoral access; performing left radial access with the patient in prone position is also a suggested option to minimize exposure of staff.

02. CCS Category III:

Until the curve of new/ active COVID 19 cases flattens, which for India, is not anticipated before July 2020 [9], it is suggested that CCS category III should be managed as for CCSC I and II. Level-1 PP should be used. After the curve flattens, it may be possible to perform invasive procedures on patients as per American College of Cardiology(ACC) guidelines, which means directly taking patients to CL for PCI for STEMI and early invasive treatment for ACS. Other countries in Stage 4 of the epidemic, or beyond would have to decide on their anticipated timeline based on advice from WHO and from the public health experts and epidemiologists.

\section{B. Infrastructural considerations and arrangements and space plans.}

'COVID 19 designated centers' for various procedures, including the $\mathrm{CL}$ should have a separate segregated corridor for movement of patients in and out of the $\mathrm{CL}$ or equivalent procedure areas. $\mathrm{A}$ separate chamber is required for donning and doffing PPE by the staff, which should be adjoining space for showering and sanitization of personnel after doffing. Personnel assisting in donning and doffing of PPE should be adequately protected. Reuse of PPEs should be prohibited. It is useful to have runners standing by outside the door of $\mathrm{CL}$ or OR for an initial few CCS category I or II cases, to cater to 
Any unforeseen and unexpected requirements that may arise during the performance of these procedures. Patients needed to wear masks and maintain a social distance of at least two meters between them. Crowding in consulting rooms and in waiting areas must be avoided. Only 1 patient should be in a consulting room at a time.

\section{Operational issues in COVID 19 'designated' and 'enabled' facilities}

- The reuse of personal protection equipment (PPE) including N95 for seeing or operating upon such asymptomatic carriers also risks the spread of infection since an asymptomatic carrier may be shedding the virus and contaminate this equipment without the user being aware of the increased risk to himself and to others. There are protocols for reuse of N95 sanitized by ultraviolet exposure.

- Since it is hard for a person to hear after donning the PPE, arrangements, and methods for communication between staff have to be worked out as part of the standard operating protocols (SOPs). For those working in a sterile environment for invasive and interventional procedures as in the $\mathrm{CL}$ or Operating rooms $(\mathrm{OR})$, the coordination between primary operators and assisting or circulating staff may pose challenges and this needs to be overcome with a pre-decided SOP.

- Because of the risk of virus exposure from aerosolization; endotracheal intubation if considered necessary is preferably performed prior to transfer to the $\mathrm{CL}$ with minimum staff $[12,13]$.

- For the same reasons, nebulization, positive pressure non-invasive ventilation, and the use of an Ambu bag should be avoided [12,13]. During CPR, closed-circuit ventilation and suctioning should be used instead of Ambu bag.

- Sanitization of objects in the work areas including equipment ( $\mathrm{CL}$, echocardiogram) controls, keyboards, mouse, remotes in $\mathrm{CL}$ as well as computer controls, transducers, doors, and doorknobs, work desk, etc. which get touched with bare hands should be performed using $70 \%$ isopropyl alcohol after use every time [13].

- Unused stuff in $\mathrm{CL}$ including cardboard boxes that hold stents or balloons must be discarded immediately after the case. Similar universal, general precautions (Appendix-B) are required
In OR $\mathrm{s}$ and all other areas such as CCU. The COVID 19 virus can survive on cardboard for prolonged periods of up to 24 hours.

- Modification of ventilation system: An airhandling unit (AHU), in a standard positive pressure ventilation system distributes airconditioned draught to different rooms, including the $\mathrm{CL}$ and its control room. The risk of cross-contamination from airborne infection is low if the staff is adequately protected with appropriate PPE $[10,12]$.

While a positive pressure room is capable of rapidly eliminating viruses from the $\mathrm{CL}$; it may contaminate other adjacent rooms. Negative pressure ventilation [14] on the other hand optimally protects adjacent areas;

However, when a temporary negative pressure is changed to positive pressure it may cause crosscontamination of adjacent areas. Further such conversion from positive to negative pressure is time- consuming and may not be available for emergent procedures at all times.

\section{Staffing for 'COVID-19 designated' facilities}

For COVI19 'designated' facilities, the suggested work plan could be that teams should work for 7 days and be in quarantine for the following 14 days. If tested negative after 14 days, they can rejoin duties or take off to meet their families. Thus 4 teams will be required.

In addition, backup arrangements for staffing are required to cover for situations where a staff member reports sick or is quarantined. In addition to institutional costs is unavoidable and has to be dealt with per institutional, departmental, and health policy rules of the region.

\section{E. Considerations for staff working in COVID 19 'enabled' and 'designated' centers}

In those on duty in areas such as COVI19 enabled or designated center CCU or other areas, including outpatients, echocardiography or non-invasive cardiology laboratories, wherever the PPEs cannot be doffed for 8 hours, because of cost of such equipment; staff have to be aware of their personal food and drink consumption prior to donning as well as of their toilet requirements. For these reasons, hygienic disposable diapers have been used by such staff on long shifts in China, prior to donning PPE. Alternatively, per local SOPs, the shifts can be divided between staff to shorten its duration. 


\section{Limitations}

The triage of patients and the categorization of patients according to CCSC is based on the history and symptoms of patients. This pre-supposes that patients will not conceal their symptoms for fear of being turned away to a 'COVID 19 designated center', as has been reported by many physicians already. Since such centers may not be centers of their choice for the special treatment that they seek; they are liable to conceal COVID-19 symptoms. In India, for example, so far, the COVID19 designated hospitals in the initial phase are only the public sector hospitals. If the public sector were to get overwhelmed, akin to what happened in the US, though such segregation of centers may not be feasible; though, learning from the experience of China [9], this should be ensured.

\section{Conclusion}

In conclusion, the battle with COVID 19 pandemic may be a protracted one worldwide as the escalating new positive cases indicate. In view of the risk to healthcare from patients and asymptomatic carriers and consequential spread to the other patients and community, it is imperative that health care systems and hospitals quickly evolve their own unique SOPs to deal with the challenge. This document provides broad guidance based on consideration of various issues related to the disease, it's the diagnosis and the health care resource limitations.

\section{Acknowledgments}

Authors would like to thank the wellness and radial intervention society

\section{Reference}

01. WHO. Coronavirus disease (COVID-2019) situation reports. Geneva- World Health Organization. 2020. Available at:

[Article] [Crossref]

02. Huang C, Wang Y, Li X, Ren L, Zhao J, Hu Y, et al. Clinical features of patients infected with 2019 novel coronavirus in Wuhan, China. Lancet. 2020;395(10223)497-506. doi: [Article] [Crossref]

03. Center for Disease Control and Prevention. Coronavirus (COVID-19). 2020. Available at: [Article] [Crossref]
04. LM Kucirka, SA Lauer, O Laeyendecker, Boon D, Lessler J. Variation in false negative rate of reverse transcriptase polymerase chain reaction-based SARS-COV-2 tests by time since. Ann Intern Med. 2020;M20-1495.

doi: [Article] [Crossref]

05. Mizumoto K, Kagaya K, Zarebski A, Chowell G. Estimating the asymptomatic proportion of coronavirus disease 2019 (COVID-19) cases on board the Diamond Princess cruise ship, Yokohama, Japan, 2020. Euro Surveill. 25(10) 2000180.

doi: [Article] [Crossref]

06. Ganyani T, Kremer C, Chen D, Torneri A, Faes C, Wallinga J, Hens N. Estimating the generation interval for COVID-19 based on symptom onset data. Euro Surveill. $2020 \mathrm{Apr} 30$; 25(17) 2000257.

doi: [Article] [Crossref]

07. Van Doremalen $\mathrm{N}$, Bushmaker $\mathrm{T}$, Morris $\mathrm{DH}$, Holbrook MG, Gamble A, Williamson BN, et al. Aerosol and surface stability of SARS-CoV-2 as compared with SARS-CoV-1. New Engl J Med. 382(16)1564-1567.

doi: [Article] [Crossref]

08. Guo YR, Cao QD, Hong ZS, Tan YY, Chen SD, Jin $\mathrm{HJ}$, et al. The origin, transmission and clinical therapies on coronavirus disease 2019 (COVID19) outbreak-an update on the status. Mil Med Res. $2020 ; 7(1) 1-10$. doi: [Article] [Crossref]

09. COVID 19 for India updates, March 24,2020, center for disease dynamics economics and policy. Available at:

[Article] [Crossref]

10. Cheng $X$. Protecting cardiologists during the COVID-19 epidemic-lessons from Wuhan, China [Internet]. Escardio org European Society of Cardiology. 2020. Available at:

[Article] [Crossref]

11. Hamon M, Pristipino C, Di Mario C, Nolan J, Ludwig J, Tubaro $M$, et al. Consensus document on the radial approach in percutaneous cardiovascular interventions- position paper by the European Association of Percutaneous Cardiovascular Interventions and Working Groups on Acute Cardiac Care** and Thrombosis of the European Society of Cardiology. Euro Intervention. 2013;8(11)12421251.

doi: [Article] [Crossref] 
12. Seto $W H$, Tsang $D$, Yung RW, Ching TY, Ng TK, Ho $M$, et al. Effectiveness of precautions against droplets and contact in prevention of nosocomial transmission of severe acute respiratory syndrome (SARS). Lancet. 2003;361(9368)1519-1520.

doi: [Article] [Crossref]

13. Szerlip M, Anwaruddin S, Aronow HD, Cohen MG, Daniels MJ, Dehghani $P$, et al. Considerations for Cardiac Catheterization Laboratory Procedures- During the COVID-19 Pandemic, Perspectives from the Society for Cardiovascular Angiography and Interventions Emerging, Leader Mentorship (SCAI ELM) Members and Graduates. Cath Cardiovasc Interven. 2020.

doi: [Article] [Crossref]

14. Chow $\Pi$, Kwan A, Lin Z, Bai W. Conversion of operating theatre from positive to negative pressure environment. J Hosp Infect. 2006;64(4)371-388.

doi: [Article] [Crossref]

\section{Appendix-A}

Level-1 PP (See text for abbreviations) Includes donning (Adapted from $\mathrm{CDC}$ ) the following as described in the Donning sequence below. The donning protocol must be displayed in the change room and $C L$ scrub areas. Donning sequence:

01 . Change into a scrub suit.

02. 4 layered cover on feet:)(i)socks(ii)legging made of non-porous fabric and plastic(iii) hospital clean slippers/ footwear (iv)shoe cover of plastic.

03. Lead apron for those working in CL. For COVID designated center, COVID designated lead apron.

04. Scrub Hands with Betadine liquid for those performing sterile procedures

05. First head cover cap of non -porous fabric to cover the head, and ears. Those with long hair choose the appropriate headcover and tie hair in a bun.

06. Wear an N95 mask

07. The second head cover cap of non -porous fabric to cover the head, neck, and ears.

08. Surgical mask.

09. Goggles and/or transparent visor (face shield) to cover face should be worn over personal
Spectacles. A face shield is to be preferred to cover sides of the face, if hooded suits are not worn.

10. Sanitize hands by rubbing with SteriliumTM

11. A Level -C (US classification) Hazmat or equivalent innovative suite (Figure 3 ) made of non-porous fabric, plastic ( \pm rubber). Should preferably have a hood to cover the head and neck. It covers the entire body including, trunk and upper and lower extremities up to legs.

OR

Wear unsterile gown with 3rd Cap of non porous fabric. Must cover head and ears and neck and prefer face shield instead of goggles if HAZMAT or equivalent hooded suit not worn.

12. 1st Sterile Gloves

13. 2nd gown: a Sterile disposable Gown made of non-porous fabric, available in a universal protection kit; for those performing or assisting in any sterile procedures.

14. 2 sterile gloves: preferably including 1 pair of latex gloves for those performing or assisting in any sterile procedures; only 1 sterile glove is adequate for those not performing sterile procedures.

Note: For those not performing sterile procedures, such as those doing outpatient, inpatient, or noninvasive work:

- Unsterile gown in Step 13 and examination gloves instead of sterile gloves may be used by those not required to perform sterile procedures.

- Specific change areas should be made available.

Doffing (Adapted from $C D C$ ) is done in a sequence reverse to Donning:

01. Hand rub (HR1) with Sterilium/methylated Spirit/70\% isopropyl alcohol

02. Remove Sterile disposable surgical gownbreakneck/back straps and discard layer3 sterile gloves.

03. HR2 with Methylated spirit or sterilium in $\mathrm{CL}$ or $\mathrm{OR} /$ procedure room (PR)

04. Remove face cover/eye goggles

05. Remove surgical mask

06. Remove the second headcover in CL/OR/PR

07. Remove Hazmat suit or equivalent and glove layer-2 
08. HR3 with Sterilium/methylated Spirit/70\% isopropyl alcohol in CL/OR/PR

09. Remove shoe covers at the doorway in the room and step out of CL/OR/PR

10. HR4 with Sterilium/methylated Spirit/70\% isopropyl alcohol

11. Remove the N95 mask (Best removed by covering the $\mathrm{N} 95$ mask with a cup-shaped container of neutral material such as melmoware, and putting the straps of the mask on the back of the container. Some centers reuse these $\mathrm{N} 95$ masks again after 4 days of dormancy)

12. Remove the first headcover

13. HR5 with Sterilium/methylated Spirit/70\% isopropyl alcohol

14. Remove lead apron for those in $\mathrm{CL}$

15. Shower, sanitize whole body and wash personal spectacles with soap and water

16. Change to clean scrubs and shoes/slippers

Level-2 PP: This differs from 'Universal protection ' in that there are 2 additional layers of protection from head to toe, as in Level 1 . The same donning and doffing sequence as in Level 1PPE can be followed. A HAZMAT suit is not mandatory; an equivalent made locally innovatively with nonporous fabric and plastic cover may suffice. Alternatively, a sterile non-porous, non-woven disposable gown can be used, on top of a plastic gown; while maintaining a triple-layer cover for head and neck hands (triple gloves) and feet.

\section{Appendix B}

General precautions for work areas, in $\mathrm{CL}$, ORs, and non-invasive cardiac laboratory, CCUs, and such other areas which are common to care in all specialties and procedures at all centers independent of CCS category and level of protection.

- Work plan and staffing should limit exposure to every single person and to as few individuals as feasible.

- For CL or sterile invasive procedures, It is preferable to do cases in a bunch and not in a staggered fashion, so that the operator and staff assisting can sanitize themselves, shower and change into personal clothes once at the end of the session.
- Use of an Ambu bag, nebulization, and positive pressure non-invasive ventilation should be avoided to minimize the risk of the spread of the virus.

- Early intubation is advisable in patients with desaturation and severe breathing difficulty.

- Frequent cleaning of equipment and furniture, including keyboards, mouse, CL controls, echocardiographic machine controls, keyboard, plugs, transducers, and doors, doorknobs should be performed using $70 \%$ isopropyl alcohol.

Unused stuff in the work area can harbor infection and must be sanitized or removed and discarded as soon as work is complete, including covers from lines, catheters, sheaths, stents, balloons, etc.

\section{Appendix-C}

\section{Appendix -C(1)}

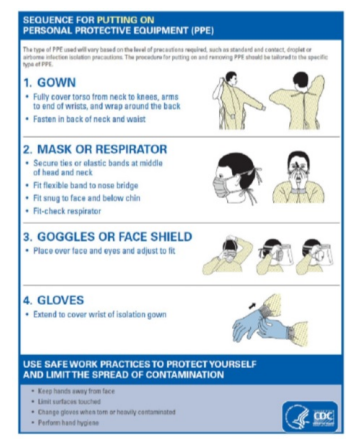

2. Appendix- C (2)

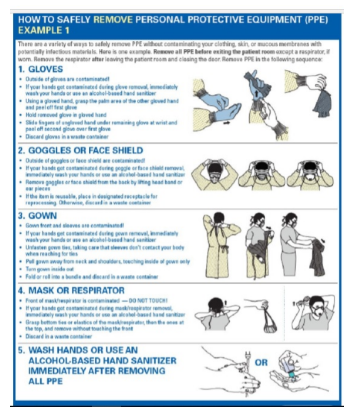

\section{Appendix -C (3)}

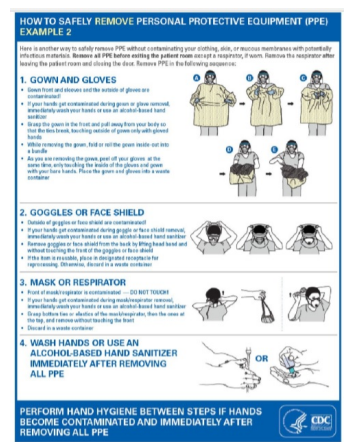

